\title{
Design-to-Manufacture Information Transfer in the Context of Solid Freeform Fabrication Technologies
}

\author{
David W. Rosen \\ Georgia Institute of Technology, United States of America
}

Key words: Solid Freeform Fabrication, Distributed Product Realization, Process Planning, Engineering Design, Decision-Based Design, Rapid Tooling Testbed

\begin{abstract}
With Solid Freeform Fabrication (SFF) technologies, the possibility of implementing a VLSI-like metaphor for design-manufacturing information transfer is enabled. That is, it has been hypothesized that a standard interchange format for SFF can be developed that enables design activity to be separated from manufacturing activity and that little additional communication between design and manufacturing organizations is necessary. In this paper, this hypothesis is explored for various points along the product design timeline. At earlier times in the design process, the requirements for a standard interchange format change as do requirements on SFF process planning. Advantages and disadvantages of early transfer of design information are presented. A system architecture and information models are discussed for enabling design-manufacturing transfers at various points in the design timeline. Examples are presented that illustrate progress toward this architecture and demonstrate the capabilities and limitations of our progress.
\end{abstract}

\section{INTRODUCTION}

Increased competition is forcing product realization to change: to become faster and to leverage facilities and expertise, wherever they may be. Imagine a product realization environment in which engineers collaboratively develop, build, and test solutions to design-manufacture problems encountered in product realization processes. In this paper, I present progress toward this vision, highlighting information requirements at 
the design-manufacture interface. This work is in the context of the Rapid Tooling TestBed (RTTB), a distributed computing environment and design methodology that incorporates rapid prototyping and rapid tooling technologies (Allen \& Rosen, 1997). The RTTB is intended to support the design of parts and molds, the selection of prototyping technologies and vendors, and the fabrication of those parts and molds. The RTTB integrates design process and fabrication process models with product models to enable a designer to instantiate an approved design process with the appropriate tasks, personnel, vendors, software, and equipment.

The purpose of this paper is to present conditions under which design representations can be transferred to a manufacturing organization. The research question that is addressed here is:

How early, and under what conditions, can design representations be transferred to manufacturing?

This problem is explored in the context of the RTTB and SFF technologies. By "transferred to manufacturing," I mean that the manufacturing organization assumes responsibility for delivering parts or product with little additional input from the designer(s). A design representation that can be transferred to manufacturing meets the objectives of a VLSI-like interface between design and manufacturing. This representation must be process independent, but must contain enough information to enable the manufacturing organization to fabricate the artifact. Since SFF technologies build parts using a large number of simple, well understood operations, it is hypothesized that process planning can be based on powerful geometric decomposition algorithms, enabling the automation of process planning and the prediction of part properties. Such a situation achieves the desired VLSIlike interface.

The problem defined by the research question can be restated more informally as "who is responsible for design-for-manufacturing (DFM)?" DFM is often difficult for mechanical parts since significant manufacturing knowledge is required to adjust part designs to aid manufacturability by a specific process. Small design changes can cause large changes in the manufacturing process or may render that process infeasible. However, if the manufacturer understands the purpose of a design and its functional requirements, then the manufacturer may be in a better position to adjust the design to facilitate manufacturing without compromising functionality. Thus, the research question asks if design representations can be developed that enable the manufacturer to perform DFM.

The baseline case is the usage of service bureaus by engineering organization for prototype parts, generally fabricated by SFF technologies. Typically, an engineer will send a STL file electronically to a service bureau, the part will be fabricated, then shipped overnight so the engineer receives it 
in 2-3 days. The STL file is a well-known standard interchange format, but it has significant disadvantages (Kumar \& Dutta, 1997).

In Figure 1, three candidate points are shown where this design-tomanufacture transfer could occur, indicated by the numbered circles (D-M Interfaces in the Figure key). The Geometric Tailoring activity indicates any design operations required to ensure manufacturability, such as adding rounds and draft to an injection molded part. Should the design or manufacturing organization be responsible for geometric tailoring? Referring back to Figure 1, it is clear that a manufacturing process and a part material must be selected prior to fabrication. Again, which organization should be responsible for doing so?

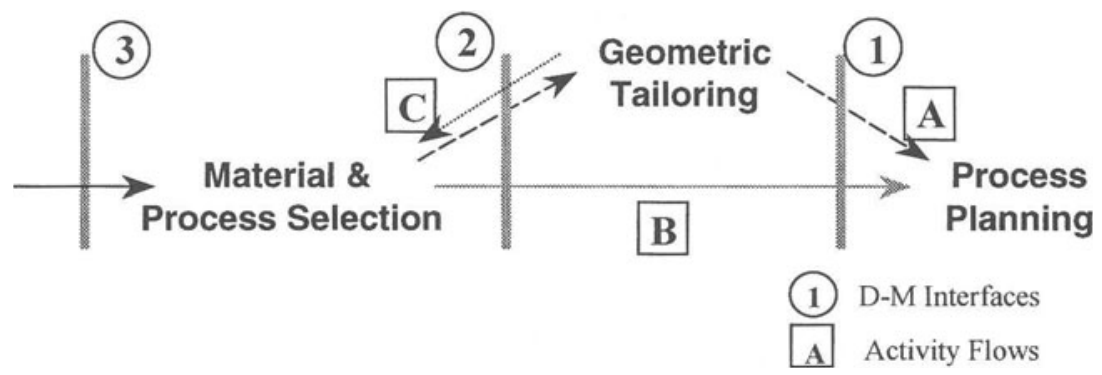

Figure 1. Design-Manufacture Interfaces and Activity Flows.

Figure 1 shows three different sequences of activities, labeled by the letters in squares. In some cases, no geometric tailoring may be necessary, indicated by flow B. For example, when a company orders a stereolithography part from a service bureau, they probably did not change the part design to facilitate the SLA process. Sometimes a geometric tailoring event is required, such as in injection molding; this is flow A. Flow $\mathrm{C}$ indicates iteration between material \& process selection and geometric tailoring. Such an iterative flow is necessary if the selection depends upon the extent or nature of part redesigns to facilitate fabrication. In the STL baseline case, transfer point 1 is used, no geometric tailoring would be performed, and the designer may or may not have specified the material or process to be used, but would have likely described his requirements verbally to the service bureau.

Three questions follow from the main research question above:

1. If the manufacturing organization selects the material and process, what information must be in the design representation to facilitate this selection? 
2. To what extent can design activity be manufacturing process independent (i.e., no DFM)?

3. Conversely, can design requirements be communicated to the manufacturing organization such that adjustments to the part to facilitate manufacture result in parts that satisfy the design requirements?

Questions 1 and 3 will be directly addressed in this paper.

Investigating design representations further, Figure 2 shows a set of design and manufacturing hierarchies along with potential places where design-manufacturing transfer occur. At present, solid and other geometric models without tolerances and surface finish specifications can be transferred, assuming that the parts are to be manufactured using SFF technologies (STL file format). We are currently researching the transfer of design representations with tolerance and surface finish specifications, along with additional requirements for SFF technologies. At higher levels of information content, it is unclear if general purpose design-manufacture transfers can be achieved, in part because there are no widely accepted languages for specifying engineering requirements.

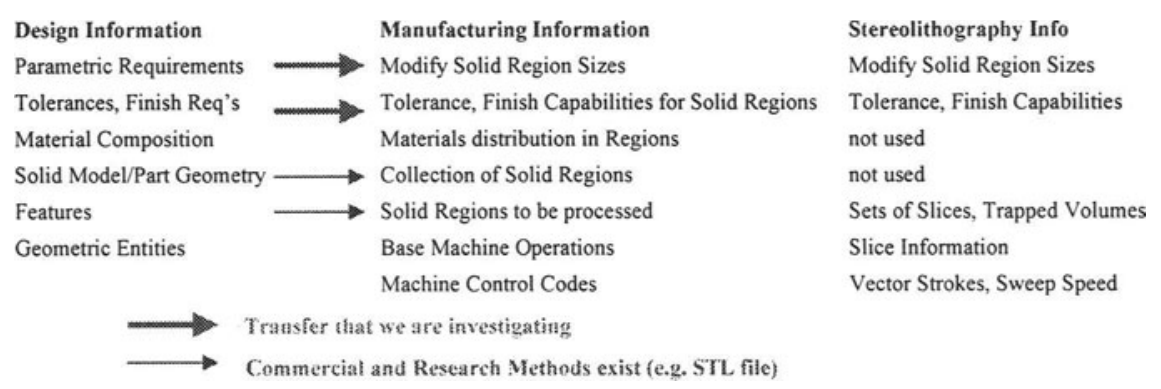

Figure 2. Information Hierarchies for Transferring Product Representations from Design to Manufacturing.

In Section 2 of this paper, the approach to the research is covered with an emphasis on the major bodies of literature underlying the research. In Section 3, the RTTB architecture is briefly described, outlining the major software modules and the distributed computing environment being constructed. Given the desired software architecture, information modeling requirements are discussed in Section 4 to support this architecture. In Section 5, three examples are presented that illustrate different designmanufacture transfer situations. These examples demonstrate one approach at achieving design-manufacture transfer at earlier points in the design process and highlight the challenges in doing so. The paper concludes with summary observations and directions of future work. 


\section{RESEARCH APPROACH}

There is a tremendous amount of literature that could be referenced in support of this paper. In the interests of brevity and focus, two areas will be emphasized, that of decision making in design and process planning for SFF technologies. Both the selections (materials and processes) in Figure 1 and the geometric tailoring activity can be modeled as decisions. Clearly material selection is a selection decision. Geometric tailoring is a multiobjective decision with dimensions as variables; this can be formulated as a multiobjective optimization problem.

\subsection{Decision-Based Design}

We use the framework of Decision-Based Design, DBD, for describing a typical product realization process. In Decision-Based Design, decisions serve as markers to identify the progression of a design from initiation to implementation to termination. The implementation of Decision-Based Design can take different forms. In mechanical engineering there is an increasing awareness that decisions made by designers could be the key element in the development of design methods that facilitate design for the life cycle and foster concurrency in the process, for example, Hazelrigg (1996), Suh, (1990), and Whitney, et al. (1988) support this notion as well. Our approach is called the Decision Support Problem (DSP) Technique (Mistree et al., 1990). It has been developed and implemented to provide support for human judgment in designing systems that can be manufactured and maintained.

The DSP Technique consists of three principal components: a design philosophy rooted in systems thinking, an approach for identifying and formulating Decision Support Problems (DSPs), and software, the DSIDES, package (Mistree et al., 1993). The DSP Technique requires that a designer implement two phases, namely, a meta-design phase and a computer-based design phase. Meta-design is accomplished through partitioning a problem into its elemental DSPs and then devising a plan of action.

Decision Support Problems provide a means for modeling decisions encountered in design and the domain specific mathematical models so built are called templates. Multiple objectives, quantified using analysis-based "hard" and insight-based "soft" information, can be modeled in the DSPs. For real-world, practical systems, all of the information for modeling systems comprehensively and accurately in the early stages of the project may not be available. However, solutions to DSP's can be used to support a 
designer's quest for a superior solution. Formulation and solution of DSPs provide a means for making the following types of decisions:

- Selection - the indication of a preference, based on multiple attributes, for one among several alternatives (Mistree et al., 1989).

- Compromise - the improvement of an alternative through modification

(Mistree et al., 1990 and Mistree, et al., 1993).

Word formulations of the Decision Support Problems are shown in Table 1. Note that each is described by a set of keywords (Given, Find, Identify, etc.) and descriptors (alternatives, attributes, importances, etc.) which form a language for describing decision problems. The math form of the compromise DSP is shown in Figure 3. There is a 1-to-1 correspondence between word and math forms of this DSP; furthermore, specific forms of bounds, constraints, and goals and deviation functions ensure that all compromise DSP's are structured in the same manner, essentially defining a mathematical language for specifying such problems. It is important to note that the compromise DSP is an extensive of goal programming methods, where the purpose is to satisfy "rigid" objectives, called constraints, and to meet "soft" objectives, called goals, as well as possible, provided significant flexibility in solving problems. Hence, the compromise DSP is not a traditional multiobjective optimization formulation.

In addition to selection and compromise decisions, combinations of decisions in coupled, hierarchical or non-hierarchical forms are possible; selection/selection, compromise/compromise and selection/compromise decisions may be coupled (Bascaran et al., 1989; Herrmann \& Allen, 1999).

Table 1. Word Formulations for Selection and Compromise Decision Support Problems

\section{Selection DSP}

Given: Alternatives from which to select.

Identify: Key attributes that influence the selection of alternatives.

Relative importances of attributes.

\section{Compromise DSP}

Given: Alternative to be improved through modification.

Find: Values of System Variables.

Values of Deviation Variables.

Rate: Alternatives with respect to each Satisfy: Goals, Constraints, Bounds attribute.

Rank: Order the alternatives in terms of Minimize: Deviation of solution from goals. preference.

\subsection{SFF Process Planning}

Currently there is a great deal of literature available for process planning of SFF technologies such as stereolithography. This literature spans from 
topics such as build process optimization, to inaccuracy prediction and correction, and support structure generation. The work presented in this paper relates to process planning issues.

\section{Given:}

An alternative to be improved through modification.

Assumptions used to model the domain of interest.

\section{Find:}

System variables values: $\quad X_{1}$

Deviation variables values:

$$
\begin{array}{ll}
\mathrm{X}_{\mathrm{i}} & \mathrm{i}=1, \ldots, \mathrm{n} \\
\mathrm{d}_{\mathrm{i}}^{-}, \mathrm{d}_{\mathrm{i}}^{+} & \mathrm{i}=1, \ldots, \mathrm{m}
\end{array}
$$

\section{Satisfy:}

Bounds: $\quad \mathrm{X}_{\mathrm{i}}^{\text {min }} \leq \mathrm{X}_{\mathrm{i}} \leq \mathrm{X}_{\mathrm{i}}^{\max }$

System constraints:

$$
\begin{array}{ll} 
& \mathrm{i}=1, \ldots, \mathrm{n} \\
\mathrm{g}_{\mathrm{i}}(\mathbf{X}) \leq 0 & \mathrm{i}=1, \ldots, \mathrm{p} \\
\mathrm{A}_{\mathrm{i}}(\mathbf{X})+\mathrm{d}_{\mathrm{i}}^{-}-\mathrm{d}_{\mathrm{i}}^{+}=\mathrm{G}_{\mathrm{i}} & \mathrm{i}=1, \ldots, \mathrm{m} \\
\mathrm{d}_{\mathrm{i}}{ }^{-}, \mathrm{d}_{\mathrm{i}}^{+} \geq 0, \mathrm{~d}_{\mathrm{i}}^{-} \cdot \mathrm{d}_{\mathrm{i}}^{+}=0 & \mathrm{i}=1, \ldots, \mathrm{m}
\end{array}
$$

System goals:

Deviation variables:

\section{Minimize:}

Archimedean deviation function: $\mathrm{Z}=\Sigma \mathrm{W}_{\mathrm{i}}\left(\mathrm{d}_{\mathrm{i}}^{-}+\mathrm{d}_{\mathrm{i}}^{+}\right)$, where $\Sigma \mathrm{W}_{\mathrm{i}}=1, \mathrm{~W}_{\mathrm{i}} \square 0$

Figure 3. Mathematical Formulation of the Compromise Decision Support Problem

Many researchers have investigated adaptive slicing of parts for layer based fabrication. The objective of adaptive slicing is to develop a slicing scheme, or method of slicing the CAD model, that meets a user-defined tolerance. This tolerance, commonly referred to as a cusp, serves as an indication of the allowable deviation between the true CAD model surface and the physical surface of the prototype. The error associated with this deviation is present in all layer-based manufacturing technologies to one degree or another and is referred to as the stairstep effect. Separately, Dolenc and Mäkelä (1994) and Tata (1995) were some of the first researchers. They adaptively sliced parts that were represented using STL files. Other researchers, including Sabourin et al. (1996), Kulkarni and Dutta (1996), and Xu et al. (1997) have presented adaptive slicing methods that slice CAD part models represented by analytical surfaces. All approaches attempt to improve the geometric accuracy of the physical prototype by calculating the appropriate layer thickness based on the local geometry of the CAD model, which will minimize the error associated with the stairstep effect to an acceptable level as defined by the cusp. The effect of the adaptive slicing method is to reduce the layer thickness in areas of high vertical curvature. Generally, this work seeks to meet cusp specifications, while minimizing the time to build the prototype.

Marsan et al. (1997) take a broad view of the overall process planning and break process planning into four steps. The first step involves entering 
design data into a Solid Builder, used to generate a B-rep solid model. The next step is orienting the solid model in the Orientation Module, based on one of the following criteria: minimum build height, minimum support contact area, maximum area of base, minimum volume of supports, or minimum average surface roughness. The supporting structure is then automatically generated and passed on to an Adaptive Slicing Module, where it is adaptively sliced to minimize the error associated with the stairstep effect. The final module is Path Planning which is currently undertaken using commercial software. The process planning system outlined supports a variety of SFF technologies.

Research at Georgia Tech has focused on developing methods to facilitate trade-offs among build time, accuracy, and surface finish goals. McClurkin and Rosen (1998) developed a computer aided build style selection (CABSS) tool that aids users in making trade-offs among these goals. Only three variables were considered: part orientation (3 discrete choices), layer thickness, and hatch spacing. Lynn-Charney and Rosen (1999) extended this work by conducting a detailed study of SLA accuracy. That research presents a method where response surfaces are used to quantify the achievable accuracy for a set of geometric tolerances applied to a variety of surface types. The four build-style variables investigated in that research were fill-overcure, hatch-overcure, sweep period, and z-level wait period. West (1999) has significantly extended this work to incorporate adaptive slicing and more accurate surface finish models. His work will be presented in the first example in Section 5.

\section{RTTB ARCHITECTURE}

The architecture of the RTTB computing environment will be outlined. The long term vision of the RTTB is a distributed computing environment that supports the design and manufacture of prototype parts. The environment should be able to reconfigure itself to adjust to changes in computing resources. Additionally, it should reconfigure the organization of its software modules in response to the nature of a specific designmanufacture problem. That is, no strict design process is hard-coded into the RTTB; the design process adjusts to the starting information and the required output. Both commercial and research SFF technologies are being supported, as well as select rapid tooling technologies to produce multiple parts through injection molding.

The RTTB integrates design process and fabrication process models with product models to enable a designer to instantiate an approved design process with the appropriate tasks and resources (Allen and Rosen, 1997). 
Selection, CAD, synthesis, analysis, and simulation software modules are integrated into the RTTB. The RTTB is implemented on top of the distributed computing environment called PRE from Sandia National Laboratory. We have developed the Fabrication Description Language (FDL) as the language in which fabrication processes can be described and process, material, and vendor capabilities can be encoded.

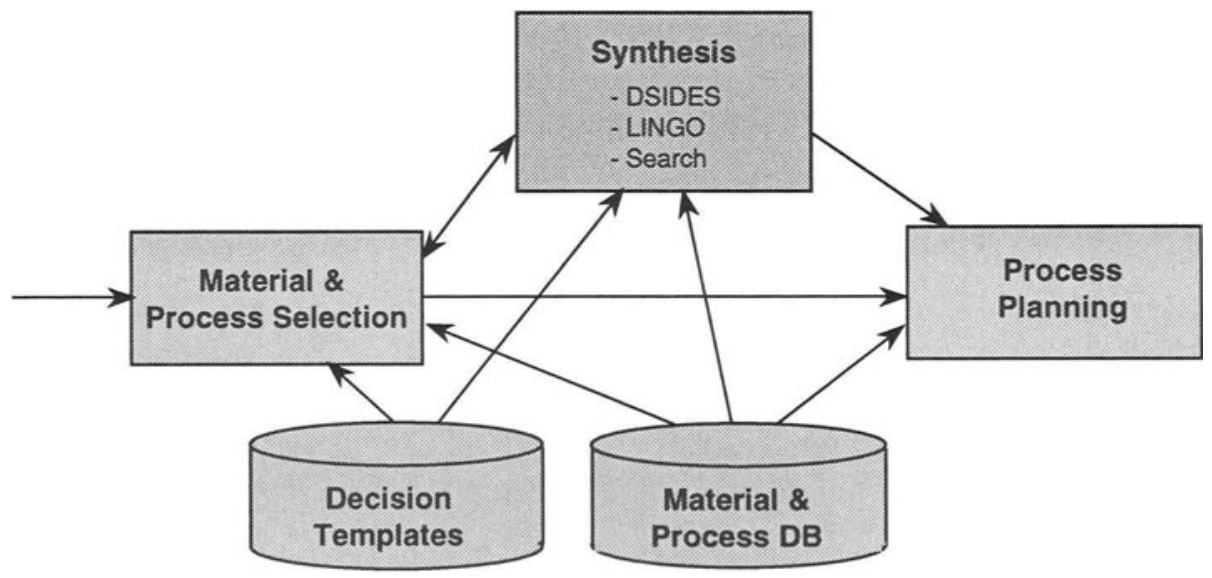

Figure 4. RTTB Architecture

To focus the presentation, only the subset of RTTB that is relevant to this paper is presented in Figure 4. Legacy codes such as ProEngineer and ANSYS have been wrapped so that they can be integrated into the RTTB. Additional software tools have been developed to perform other tasks and have been integrated as well. A more complete description of the RTTB architecture, focusing on its distributed computing nature, is provided in reference (Gerhard et al., 1999). To initiate RTTB operation, the designer provides a CAD model of his part, tolerances and surface finish specifications, preferences for prototype part characteristics (accuracy, finish, build time). Optionally, the designer may specify performance specifications, desired part characteristics, preferences for materials or fabrication processes, and preferences on other specifications and characteristics.

A web-based tool has been written in Java for coupled material and process selection (Herrmann \& Allen 1999). Material property and SFF process capability descriptions reside in a relational database and provide this information to the selection tool. A process planning tool was written in $\mathrm{C}++$ for the stereolithography process (West, 1999). Input to process 
planning consists of an ACIS solid part model with tolerances and surface finishes specified.

The synthesis module performs geometric tailoring. Two multiobjective optimization codes are currently integrated, DSIDES (Mistree et al., 1993) and LINGO (Nemhauser \& Wolsey, 1988), as well as an exhaustive search code. At present, there is no elegant method of formulating and solving geometric tailoring problems, given only the RTTB input information from the designer. Compromise DSP templates must be constructed by hand and analysis codes must be written and integrated into one of the optimization codes. However, there is some promise of generating templates automatically, given the RTTB input information (see Section 4). Information can flow along any of the arrows in Figure 4, so that iteration among the major RTTB modules is possible. The selection and synthesis modules can be coupled to enable the solution of coupled selectioncompromise Decision Support Problems.

With the RTTB architecture outlined, the stage is set to investigate the information modeling requirements to enable the RTTB to function.

\section{INFORMATION MODELING}

Information model requirements depend upon the design-manufacture transfer point, as presented in Figure 1. The earlier in the design process that the transfer is to occur, the greater the variety of information that needs to be transferred. Conversely, the later in the process that transfer occurs, the greater is the level of detail of information. In this section, some information modeling issues are raised and our approach to modeling a variety of information types is presented.

\subsection{Information Requirements}

Simply put, the information requirements for the RTTB include that information required to perform the activities in Figure 1. Nominally for process planning, a geometric part representation is required, as are a target fabrication process and material choice. The geometric model may be a solid CAD model, an STL file, or a part model in a data exchange language such as IGES or STEP. The advantages and disadvantages of each choice have been extensively debated, but this debate will not be addressed here (Kumar \& Dutta, 1997). However, a widely held requirement is that this representation must be process independent. In Figure 2, several levels of geometric model decomposition are included (regions to be processes and base machine operations). It is not clear if any process independent 
decompositions exist, although for most commercial SFF technologies, decomposing a geometric model into slices can be accomplished without much process-specific information as mentioned in Section 2.2.

Our approach to transferring information to process planning is to use a solid model that is annotated with tolerances, datums, and surface finish specifications. We use the ACIS solid modeling kernel to facilitate our research, but Parasolids or other solid modeling would work equally well. Additionally, the STEP data exchange standard would be an equally good (perhaps better) choice.

Information requirements for geometric tailoring are much more extensive than for process planning. Again, both a target fabrication process and material are required, but it is also necessary to specify the design freedom allowed by the designer. A general solution to the specification of design requirements does not seem to be available; approaches to this problem are application or domain-specific.

Our approach to this issue is to utilize the Decision Support Problems as the framework for specifying design requirements to the manufacturer. Furthermore, the class of fabrication processes and materials under consideration is limited to SFF technologies. In this manner, problem descriptions can be limited to requirements on a limited set of part properties and these can be readily related to fabrication process capabilities and material characteristics. Process capabilities and material characteristics can be stored in a database, then made available when needed. For example, when a part material needs to be selected, designer requirements on part strength, durability, and weight, plus requirements on lead time and cost, can be compared to the characteristics of known materials and the selected fabrication process. This comparison is facilitated by treating process and material characteristics as attributes in a Selection DSP template. This will be explained further in the next sub-section.

\subsection{Design Representations}

As stated previously, our design representation for transfer to process planning consists of an ACIS solid model with tolerance, datum, and surface finish annotations. These annotations are implemented as user-defined attributes in ACIS and are attached to the topological faces in an ACIS model. This is sufficient as input to process planning, provided that the process planning module does not need material composition (of multiple materials) information. Since most of our target processes are commercial SFF technologies that process only one material at a time, material compositions have not been investigated. Our process planner can incor- 
porate tolerance and surface finish requirements since these requirements can be evaluated using detailed process information (see Section 4.3).

Design representations for input to material \& process selection and geometric tailoring are much more complicated. We utilize DSP templates as the information modeling medium for describing design requirements to the manufacturer. We have formulated generic templates for material selection, for process selection, and for coupled material-process selection. For geometric tailoring, we have developed several domain-specific templates for gears and gear trains using a general language of material and process attributes.

As one example, the process selection DSP is shown in Figure 5. Note that there is a one-to-one-correspondence to the general word formulation of the selection DSP of Table 1. Since all fabrication processes and materials are represented in a database, it is straightforward to update our set of alternatives if new processes are developed or capabilities are improved. Not all attributes are included in this list, but our database contains more than 30. The idea is that the designer will always select from this list those attributes that are relevant to his problem, then specify their relative importances. Additionally, the designer/manufacturer can specify the alternatives that should be considered, rather than including all in the selection problem. In this manner, the designer can communicate problem requirements to the manufacturer using the common problem description language provided by the DSP templates. A summary of the information sources for selection problems is given in Table 2 .

Given: SLA-250, SLA-3500, SLA-5000, SLA-7000, SLS Sinterstation 2500, SLS RapidSteel, FDM 2000, Actua 2100, Zcorp 4206.

Identify: Key attributes from Accuracy, Surface Finish, Cost, Build Time, Post-Processing Time, Detail Capability.

Target values for each attribute.

Relative importances of attributes.

Rate: Alternatives with respect to each attribute.

Rank: Order the alternatives in terms of preference.

Figure 5. Word Formulation for the Process Selection DSP.

Table 2. Information Sources for Selection.

Designer Specified

CAD Part Model

Tolerance and Finish Specs.

Target Values for Material Properties and

Process Capabilities (Selection Attributes)

Preferences on Selection Attributes
RTTB System Provided

Material Properties

SFF Fabrication Processes \& Capabilities

Ability to Rate Alternatives vs. Attributes

Method and Software for Solving Selection

Problem 
For geometric tailoring, templates can be developed for specific types of problems, such as gear design, then applied to many different situations as needed. All templates are instantiations of the mathematical form of the compromise DSP from Figure 3. Users can adjust the problem definition by specifying importances on goals, converting a goal to a constraint, converting a constraint to a goal, and in other ways. However, it is not clear if a general geometric tailoring decision template can be formulated since domain specific analysis models are usually needed. For example, an analysis model for gear teeth strength is only applicable to gear design, but is irrelevant for cam-mechanism design.

How can designers communicate design freedoms to manufacturers so that the manufacturer can adjust the part without compromising designer requirements? Conceivably, analysis models, compromise DSP goals and constraints, and relative importances of goals can be communicated to the manufacturer to describe design requirements and specify freedoms the manufacturer has in adjusting the part design. With the general mathematical form of the compromise DSP, the structure of goals, constraints, system variables, bounds, and deviation functions has been specified, providing a language for defining decision problems. However, no general, computable format for this information has been developed. An interesting idea is to encode this information in Dynamically Linked Libraries and provide the protocol for extracting needed information (Storti et al., 1999).

A summary of the information sources for geometric tailoring problems is given in Table 3. Note that at present the designer has a much higher burden, as compared to selection problems.

Table 3. Information Sources for Geometric Tailoring

\begin{tabular}{ll}
\hline Designer Specified & RTTB System Provided \\
\hline CAD Part Model & Material Properties \\
$\begin{array}{l}\text { Tolerance and Finish Specs. } \\
\text { Performance Specs. (strength, weight, etc.) }\end{array}$ & SFF Fabrication Processes \& Capabilities \\
$\begin{array}{l}\text { Analysis Models (embodied in a software } \\
\text { module) }\end{array}$ & \\
$\begin{array}{l}\text { Compromise DSP Template that Specifies } \\
\text { Variables, Constraints, and Goals }\end{array}$ & $\begin{array}{l}\text { Method and Software for Solving Tailoring } \\
\text { Preferences on Specifications }\end{array}$ \\
\hline
\end{tabular}

\subsection{Manufacturing Process Information}

Manufacturing process information of two broad types is needed to support the engineering activities of Figure 1:

- Detailed manufacturing process information and models to support process planning and geometric tailoring, and 
- Simplified measures of process capability to support process selection and some geometric tailoring.

Each will be discussed in this subsection.

\subsubsection{Detailed Manufacturing Models}

Broadly, two types of information are critical to aiding process planning and geometric tailoring, analytical models for computing process capabilities and empirical models. Since we are supporting the stereolithography process, we have opted to utilize empirical models, rather than investigating the photo-chemistry basis of stereolithography. To date, we have developed three sets of empirical models for our SLA-250 machine:

- Geometric tolerance capability as a function of part orientation, scanning variables, and recoat variables;

- Surface finish capability as a function of part orientation, layer thickness, and surface type;

- Build time as a function of part orientation, layer thickness, and scanning and recoat variables.

More extensive and complete models are needed and can be developed, but these models are representative of the information types required for process planning and geometric tailoring. Each model will be briefly described.

Empirical models for accuracy were developed through extensive designof-experiment studies to determine quantitatively the relationships between SLA-250 variables and geometric tolerance capabilities. Response Surface Methodology (RSM) comprises mathematical and statistical techniques to enable the construction of approximation models (Myers \& Montgomery, 1995). RSM allows for a better understanding of the relationships between the inputs and the response, in this case between the build process variables and build goals, that can be written in the form of a polynomial function describing a surface, such as Equation 1. We use second order response surfaces in this work; $\mathrm{k}=2$.

$$
y=b_{0}+\sum_{i=1}^{k} b_{1} x_{i}+\sum_{i=1}^{k} b_{1} x_{i}^{2}+\sum_{i=1}^{k} \sum_{j=1, j \neq j}^{k} b_{1} x_{i} x_{j}
$$

Response surfaces are useful when detailed theoretical knowledge of the model does not exist, but the relationship between the input variables and the output variable is assumed to be smooth. This is the case for SLA machines. For instance, theoretical equations that give accuracy as a function of layer thickness and hatch spacing do not exist, but accuracy is likely to change continuously as layer thickness and hatch spacing are varied. 
The accuracy models used in this work come directly from the response surface models developed by Lynn-Charney \& Rosen (1999). By using a set of response surface models that relate four build process variables (hatch and fill overcure, sweep period, and z-level wait) with a specific type of geometric tolerance and a surface type, the obtainable accuracy for that tolerance may be predicted. Six types of geometric tolerances were considered in this work: positional, flatness, parallelism, perpendicularity, concentricity, and circularity. A total of thirty six different response surfaces were developed based on the type of surface, the orientation of that surface, and the type of geometrical tolerance. Given the tolerance type, orientation of the surface to which the tolerance is annotated, and the values of the layer and recoat variables, predictions of the achievable accuracy for every geometric tolerance can be made. One of the response surfaces for parallelism is shown in Figure 6. Deviation from perfect parallel planar surfaces is shown as a function of sweep period and z-level wait. As can be seen, deviation is mostly dependent upon sweep period; as sweep period decreases (sweep blade moves faster), deviations increase significantly.

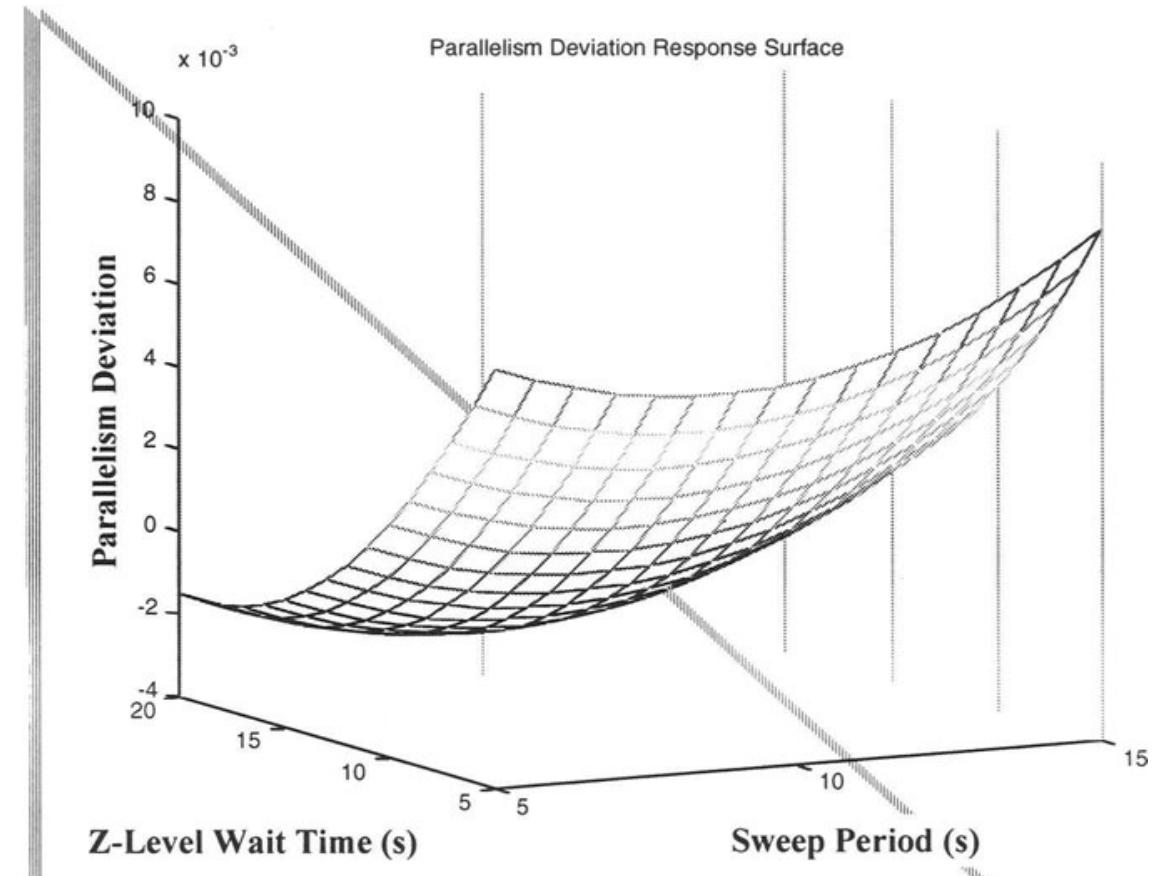

Figure 6. Parallelism Tolerance Response Surface 
Surface finish capabilities may be predicted using a set of empirical roughness models (West, 1999) that are functions of layer thickness and surface orientation. In other research, Reeves \& Cobb (1997) collected data in which the surface finish of a planar surface is measured for a series of different orientations. Similar experiments were performed in this work, but instead of associating the surface finish with the orientation, the cusp (which is a function of both layer thickness and orientation) is associated with the surface finish (roughness) as shown in the plots of Figure 7. In this manner the surface finish corresponding to a given cusp and layer thickness can be predicted for both planar and curved surfaces. Since surfaces are always composed of multiple layers, some of which may be at a different layer thickness, the average surface finish is used. Given the slice scheme for a CAD model, and the knowledge of the extents of the surfaces in the slice scheme, predictions of the surface finish may be made for every surface in the model.

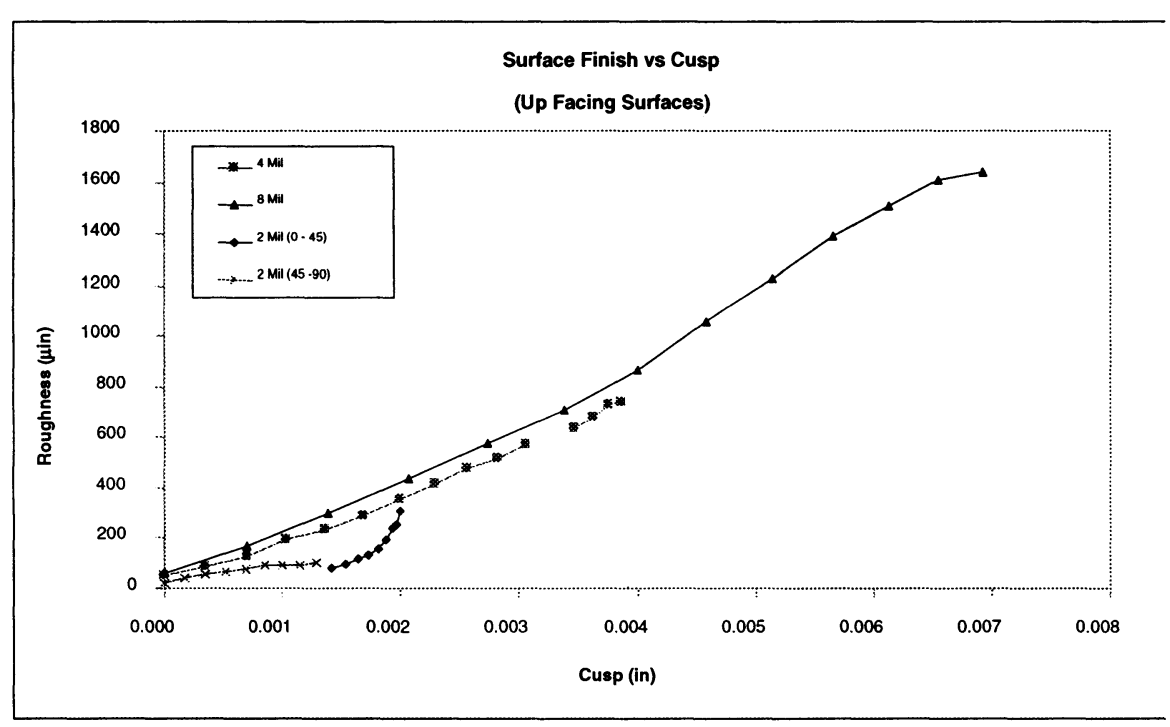

Figure 7. Surface Finish Empirical Model

In a similar manner, response surfaces were developed to predict build time. As part of our process planning method, part geometric models are sliced, enabling the slices to be used in build time prediction. Response surfaces were developed for different types of SLA scan vectors as functions of layer thickness and areas to be scanned. Again, extensive experiments were performed and actual scan times measured. Separate response surfaces were developed for border, hatch, and fill vectors. One response surface for hatch vectors is shown in Figure 8. 


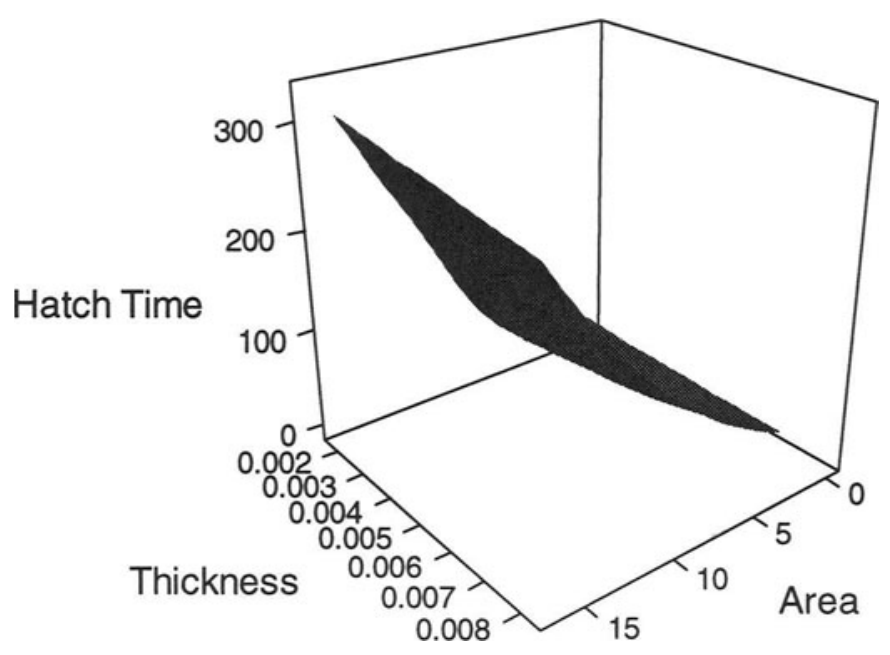

Figure 8. Build Time Response Surface for Hatch Vectors

\subsubsection{Simplified Measures}

To support material and process selection, and to enable some geometric tailoring, simplified measures of material properties and process capabilities are needed. We utilize information of the type supplied by machine vendors and MSDS material sheets, where for example, SLA-250 accuracy is given as $0.003 / 5$ in. +0.001 in. per additional inch. As a result, measures are given by individual values, ranges, or ratios.

\begin{tabular}{|l|l|}
\hline MACHINE & VENDOR \\
Machine_name $=$ SLA250 & Company_name =3D Systems Inc. \\
Technique_name=SLA & Address_Street $=26081$ Avenue Hall \\
Build_time $=20$ & Address_City = Valencia \\
Tolerance $=0.001,0.002$ & Address_State = California \\
Accuracy $=0.005$ & Address_ZipNum =91355 \\
Detail_capability=HIGH & Address_Country $=$ USA \\
Surface_finish=HIGH & PhoneNum $=805-295-5600$ \\
Support=NEED & FaxNum $=805-257-1200$ \\
Part_removal_time=45 & Email=webmaster@3dsystems.com \\
Material_name=SL5220, SL5510, SL5520 & WebSize = www.3dsystems.com \\
Vendor_name $=$ 3D Systems Inc. & Contact_person= John Smith \\
\hline
\end{tabular}

Figure 9. SLA-250 and 3D Systems (vendor) FDL Files

We have developed a simple language, called Fabrication Description Language (FDL), to describe material, manufacturing process, and vendor 
properties, capabilities, and limitations. We are extending the language to also represent manufacturing processes, the sequence of operations required to fabricate a part. Figure 9 shows example SLA-250 and 3D Systems FDL files. There are three main entities in FDL: Machine, Material, and Vendor. Additionally, there are many other entities that represent attribute values (range, ratio, list, etc.).

\section{DESIGN-MANUFACTURE TRANSFERS AND ACTIVITY FLOWS}

As shown in Figure 1, three possible design-manufacture transfer points exist in the engineering practice under investigation here. Similarly, three different activity flows are possible. In this Section, three examples will be presented to illustrate several different combinations of transfers and activity flows. Broadly, the purpose of these examples is to demonstrate one approach to enabling design-manufacture transfer before design-formanufacture has been completed. Each example is meant to illustrate some DFM capabilities that have been developed for the RTTB and highlight the information required for these capabilities.

In the first example, only the process planning activity is investigated, indicating design-to-manufacture transfer at point 1 . Either activity flow $\mathrm{A}$ or B is consistent with this example. In the second example, the geometric tailoring activity is present, indicating design-manufacture transfer at point 2 and activity flow A. It is assumed that a material and process have already been selected. In the final example, iteration among the material \& process selection and geometric tailoring activities is illustrated, indicating designmanufacturing transfer point 3 and activity flow $C$.

Two different parts are used in this section. For the first example, a bracket with some complex surfaces is used since process planning is nontrivial here. In the final two examples, a ring gear from a planetary gear train is used. Process planning for a gear such as this is straightforward, given the information of the first example.

\subsection{Process Planning with Tolerances and Finish Specifications}

The first example to be presented focuses on the process planning activity in Figure 1. Information being transferred from design to manufacturing includes solid geometry, tolerances, and surface finish specifications. Prior to process planning, a fabrication process and material were selected; they are the SLA-250 stereolithography machine and SOMOS 
7110 resin. After briefly presenting the process planning method, the results are discussed of process planning on the part shown in Figure 10.

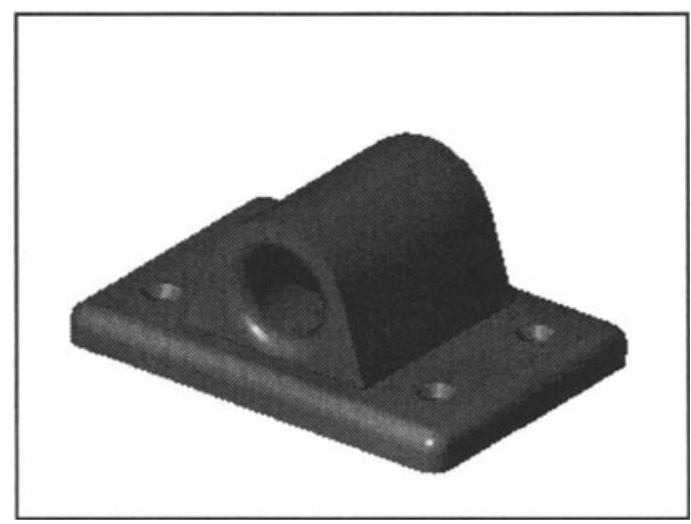

Figure 10. Bracket for the Process Planning Example

Our process planning method is intended to achieve the desired balance among the three build goals of accuracy, surface finish, and build time. This is performed by formulating and solving a Compromise DSP, where process variables are varied and an appropriate trade-off among the three build goals is achieved. The word formulation of the DSP for process planning is shown in Figure 11. The system variables consist of two part variables: the slice scheme and the orientation, two layer variables: hatch and fill overcure, and two recoat variables: sweep period and z-level wait. Part variables pertain to the build variables directly associated with the part. Layer variables pertain to the build variables that control how each layer is solidified in the vat of resin. Recoat variables pertain to the build variables that control how a new layer of resin is redeposited over the previously solidified layer. There are two constraints that are taken into consideration as well, the presence of large horizontal planes and the presence of support structures. The goals in the problem formulation consist of surface finish, accuracy, and build time.

At the heart of our process planning method is a series of three modules for orienting the part in the SLA machine, slicing the part, and selecting process variable values. All three modules are specific to the stereolithography process, although determining part orientation and slicing strategies are required in all layer-based fabrication processes. 


\section{GIVEN:}

- CAD Model

- Surface Finish Specifications

- Geometric Tolerances

FIND:

System Variables:

Part Variables:

Set of Layer Thicknesses

Part Orientation

Layer Variables:

Hatch Overcure

Fill Overcure

Recoat Variables:

Sweep Period

Z-Level Wait

SATISFY:

Goals: Surface Finish Specifications Geometric Tolerances

Minimize Build time
- Build Time target value

- SLA-250, SOMOS 7110 resin

- Goal preferences as weights

Deviation Variables: deviation of goal from target Aggregate deviation of Finish

Aggregate deviation of Accuracy

Deviation from Build Time Constraints: Large Horizontal Planes
Support Structures

MINIMIZE:

Deviation Function: Weighted sum of Goal Deviations

Figure 11. Process Planning Word Formulation

The selection of the best process plan is made by investigating the goal achievement of each of the three goals as indicated by the deviation values as well as the predicted values for accuracy, surface finish, and build time. In this formulation, the input is an ACIS based CAD model (.SAT file) with tolerances and surface finishes specified. Additionally, the user must supply measures of goal preferences. Generally, several scenarios (sets of preferences) are provided so that the user can better discern the trade-offs being made. Simple weights are used here, where the weights for accuracy, finish, and build time must sum to one. In this example problem, one set of goal preferences is investigated in depth and the results of several different sets of goal preferences are discussed briefly.

The set of layer thicknesses used for this example problem is 2 , 4, and 8 mils, which are typical values for a SLA-250. The CAD model shown in Figure 10 is a bracket and provides a variety of interesting geometric shapes and surfaces. Four surfaces of this part are deemed to be critical. The inner cylindrical surface is to have a 2.5 micron surface finish. The two rounded edges on either side of the cylindrical surface have a surface finish requirement of 3.8 micron. The down facing planar surface at the base of the bracket is to have a 3.8 micron surface finish. The remaining surfaces are set to a default surface finish of 7.6 micron. Additionally, three geometric tolerances are specified. A cylindrical tolerance $(0.0762 \mathrm{~mm})$ is applied to the inner cylindrical surface. A parallelism tolerance $(0.1016$ 
$\mathrm{mm}$ ) is applied between the down facing planar surface at the base of the bracket and the top up facing planar surface, and a flatness tolerance $(0.1016$ $\mathrm{mm}$ ) is also used on the down facing planar surface at the base of the bracket. Six scenarios of goal weights were specified by the user and are shown in Table 4. Note that the weights in a scenario must sum to one.

Table 4. Scenarios for Example 1

\begin{tabular}{ccccccc}
\hline Goal & Scen. 1 & Scen. 2 & Scen. 3 & Scen. 4 & Scen. 5 & Scen. 6 \\
\hline Accuracy & 0.9 & 0.05 & 0.05 & 0.6 & 0.2 & 0.2 \\
Surface Finish & 0.05 & 0.9 & 0.05 & 0.2 & 0.6 & 0.2 \\
Build Time & 0.05 & 0.05 & 0.9 & 0.2 & 0.2 & 0.6 \\
& & & & & & \\
\hline
\end{tabular}

Given this problem and the process planning method, the solution is computed by an exhaustive search method. Table 5 provides an overview of the resulting deviations for each of the different scenarios. The solutions that are shown in the table represent the process plans with the lowest overall deviations for the given goal preferences.

Table 5. Process Planning Results

\begin{tabular}{cccccc}
\hline Scenario & $\begin{array}{c}\text { Weighting of Goals } \\
\text { (AC, SF, BT) }\end{array}$ & $\begin{array}{c}\text { Overall } \\
\text { Dev. }\end{array}$ & $\begin{array}{c}\text { Accuracy } \\
\text { Dev. }\end{array}$ & $\begin{array}{c}\text { Surface } \\
\text { Finish Dev. }\end{array}$ & $\begin{array}{c}\text { Build } \\
\text { Time Dev. }\end{array}$ \\
\hline 1 & $(0.90,0.05,0.05)$ & 0.30 & 0.31 & 0.07 & 0.21 \\
2 & $(0.05,0.90,0.05)$ & 0.04 & 0.31 & 0.01 & 0.32 \\
3 & $(0.05,0.05,0.90)$ & 0.07 & 0.68 & 0.36 & 0.02 \\
4 & $(0.60,0.20,0.20)$ & 0.24 & 0.31 & 0.09 & 0.19 \\
5 & $(0.20,0.60,0.20)$ & 0.13 & 0.31 & 0.01 & 0.30 \\
6 & $(0.20,0.20,0.60)$ & 0.15 & 0.31 & 0.23 & 0.07 \\
\hline
\end{tabular}

As can be seen from the table, the weighting of the build goals has some affect on the resulting deviations. There are significant tradeoffs being made between the surface finish and build time goals, as one would expect. However, the accuracy goal does not appear to be significantly influenced by either of the other goals. Comparison of scenario 6 with scenario 3 provides the best example of the tradeoffs being made between the build time and surface finish goal. Both trials have a weighting scheme with the build time goal having the highest goal preference $(0.90$ for scenario 3 and 0.60 for scenario 6). The build time deviation is slightly higher for scenario 6 yet at the same time the surface finish deviation is also slightly lower. Thus it is evident that there is some degree of tradeoff being made between the build time and surface finish goal.

Although the goal deviations provide a summary assessment of a process plan, more meaningful information can be gained by examining the actual 
predictions of the surface and accuracy tolerances. Figure 12 shows the output from the process planning software for scenario 5, with all accuracy and surface finish requirements listed as well as their predicted values. Face 2 represents the inner cylindrical surface, while faces 0 and 3 represent the rounded edges on either side of that cylindrical surface. Thus in this process plan, most of the critical surfaces are meeting the specified surface finish requirements. Figure 13 provides a graphical representation of the slicing scheme for the process plan of trial 5. The darkest shading represents areas where the model is to be built with an 8 mil layer thickness while the lighter shading represents 4 and 2 mil layer thickness. As one would expect, the rounded edges on the bracket require the use of a smaller layer thickness to meet the surface finish requirements. By investigating the slicing scheme and the surface finish and accuracy predictions, a much better understanding of the given process plan may be developed. Armed with this type of information, the user can make much more informed decisions as to what process plan should be ultimately used for the fabrication of the prototype.

\begin{tabular}{ccccc}
\hline \multicolumn{2}{c}{ Accuracy Results: } & & & \\
Tol \# & Face \# & Tol Type & Desired Value $(\mathbf{m m})$ & Actual Value $(\mathbf{m m})$ \\
0 & 2 & Cylindrical & 0.0762 & 0.0762 \\
1 & 19 & Parallelism & 0.1016 & 0.381 \\
2 & 33 & Flatness & 0.1016 & 0.152
\end{tabular}

Surface Finish Results:

\begin{tabular}{ccc} 
Face \# & Desired Value (micron) & Actual Value (micron) \\
0 & 3.8 & 5.05 \\
1 & 7.6 & 4.34 \\
2 & 2.5 & 1.3 \\
3 & 3.8 & 2.64 \\
4 & 7.6 & 0.13 \\
5 & 7.6 & 6.05 \\
6 & 7.6 & 1.24 \\
$\ldots$ & & \\
31 & 7.6 & 1.24 \\
32 & 7.6 & 1.55 \\
33 & 3.8 & 1.24 \\
\hline
\end{tabular}

Figure 12. Results of Scenario 5

\subsection{Geometric Tailoring Example}

For the next two example problems, the ring gear of the transmission of a cordless drill is investigated (Connor et al., 1999). The function of the transmission in the drill is to transmit power from the motor to the chuck and its associated planetary gearing. One stage of the planetary gear train is 
shown in Figure 14. As shown, the planetary gear train consists of a sun (2), an outer frame ring (1), and a number of planets (3).

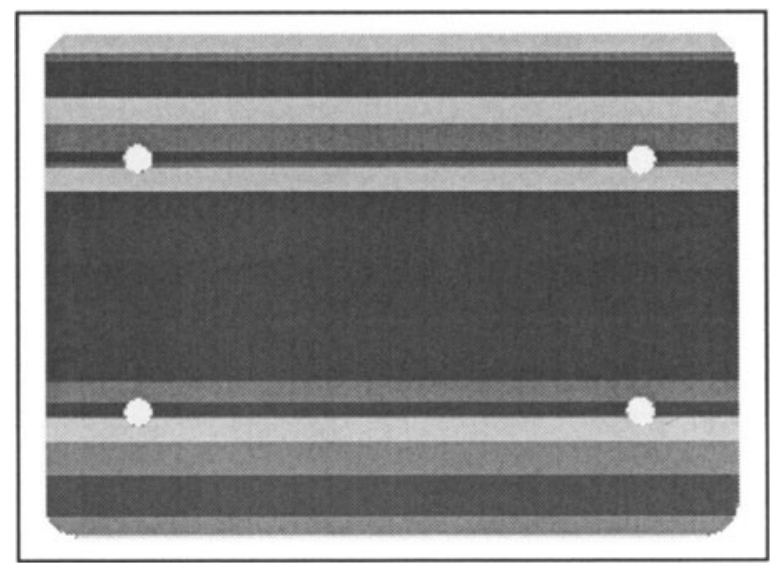

Figure 13. Slice Scheme for Scenario 5

1. Outer Frame Ring

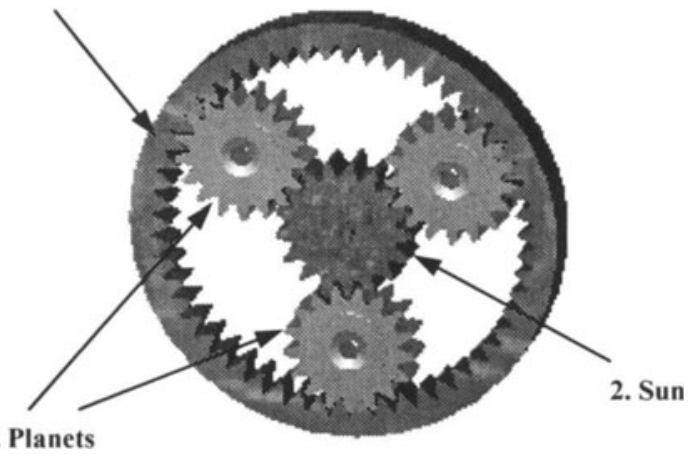

Figure 14. Planetary Gear Train - Subject of Examples 2 and 3

The purpose of this example is to investigate the geometric tailoring activity, illustrating typical decisions, and highlighting the information needed and the types of information produced. The key idea underlying geometric tailoring is that a part design must be adjusted for a prototyping technology such that some of the prototype's properties mimic those of the production part as well as possible. In this case, the production ring gear is nylon and injection molded. The designer desires a prototype gear, due to 
time constraints, that is fabricated on a SLA-250 using SL-5170 resin. The challenge is to adjust the dimensions and number of teeth of the prototype gear such that the same speed reduction is achieved and the teeth have the same bending strength as the production gear.

In the configuration assumed, the diametral pitch of the ring gear is 40 teeth/in, the face width is 0.11 in, and the number of gear teeth is 48 . A maximum of $10 \%$ error is allowed in these dimensions for the final prototyped part. This error allows for redesign of the gear, without requiring redesign of the drill casing. The elastic modulus of nylon is assumed to be $300 \mathrm{ksi}$ and the density is $0.040 \mathrm{lbs} / \mathrm{in}^{\wedge} 3$ (weight $=0.005 \mathrm{lb}$ ). The nylon gears are considered to exhibit fairly high accuracy and very good surface finish. Gear teeth must be designed to endure repeated loading, which results in bending stress. Bending failure normally occurs when the stress on a tooth exceeds the bending strength of the material of which the gear is made. The maximum load on the gear teeth is assumed to be $6.875 \mathrm{lb}$ at a maximum working speed of $10,000 \mathrm{rpm}$ and with four planet gears. The compromise DSP word formulation for this geometric tailoring problem is shown in Figure 15.

\section{GIVEN:}

- Parametrized CAD Model of Ring Gear

- Target values for variables

- Material Properties

- Target value for gear weight

- Goal preferences as weights

FIND:

System Variables:

Diametral Pitch

Face Width

Number of Teeth

\section{SATISFY:}

Goals: Meet target weight $(0.005 \mathrm{lb}$. $) \quad$ Constraint: Bending Stress

Meet target diametral pitch (40 teeth/in.)

Meet target face width $(0.11$ in.)

Meet target number of teeth (48)

MINIMIZE:

Deviation Function: Weighted sum of Goal Deviations

Figure 15. Geometric Tailoring Word Formulation

This problem was mathematically formulated, then solved using both the LINGO optimization code and an exhaustive search using a Matlab program. The solution for goal preferences of weight $=0.45$, diametral pitch $=0.22$, face width $=0.11$, and number of teeth $=0.22$ is shown in Table 6 . For this solution, only the diametral pitch and number of teeth goals were met. Due to the lower yield stress of SLA resin, the face width increased slightly. 
Also, due to differences in material properties and the face width, the gear weight was slightly greater than the production nylon gear. If preferences for the goals were changed, it is likely that different variations of system variable values would have been produced.

Table 6. Solution to the Geometric Tailoring, Ring Gear Problem

\begin{tabular}{cccc}
\hline $\begin{array}{c}\text { System Variable: } \\
\text { Value: }\end{array}$ & $\begin{array}{c}\text { Diametral Pitch } \\
40 \text { teeth/in. }\end{array}$ & $\begin{array}{l}\text { Number of Teeth } \\
48\end{array}$ & $\begin{array}{c}\text { Face Width } \\
0.111 \mathrm{in} .\end{array}$ \\
\hline Stress Constraint & \multicolumn{1}{c}{$15,498 \mathrm{psi}<15,500$ allowable } & \\
\hline Weight Goal: & Weight $=0.0051 \mathrm{lb}$ & Deviation: $0.0001 \mathrm{lb}$ & \\
\hline Deviation Function Value: & \multicolumn{3}{c}{0.0002} \\
\end{tabular}

\subsection{Coupled Selection and Geometric Tailoring Example}

The previous example can be generalized in several ways; of interest here is the integration of material and process selection into the geometric tailoring method. In the previous example, it was assumed that the SLA-250 and SL-5170 resin should be used to fabricate the ring gear prototype. However, it is desirable to consider alternatives before settling on a particular fabrication process and material. A coupled selection-selectioncompromise decision problem is illustrated here to integrate the decisions surrounding the prototype. This example demonstrates that a manufacturer can successfully select a fabrication process and material, then perform design-for-manufacture, provided that the designer quantitatively describes the design freedom allowed the manufacturer.

Stated concisely, the objective in this example is to select the fabrication process and material that enables the prototype ring gear to have desired production-representative properties. Problem requirements remain the same as in the previous problem: the ring gear is to enable a specific speed reduction, gear teeth should be strong enough, and the gear must maintain dimensions as close to production dimensions as possible. For our purposes here, three stereolithography machines will be considered and six resins (material choices) will be considered. The machines are: SLA-250, SLA3500, and SLA-5000. The resins are: SL5170, SL5210, SL5190, SL5510, SL5520, SL5195. Additional considerations upon which machine and resin selections are based include: accuracy, time, cost, surface finish, detail capability, and material properties of elastic modulus and density.

The word formulation of the coupled selection-selection-compromise decision problem is shown in Figure 16. In order to solve this problem, both additional information and the ability to solve a mixed-discrete, multiobjective optimization problem are needed. As opposed to the 
information level for process planning, the information level on process capabilities for evaluating accuracy, finish, and time are not nearly as stringent. In other words, it is possible to make good choices for fabrication process and material without detailed process capability information. In our work, we have used intervals, fuzzy sets, and crisp numbers to describe process capability and material properties (see Section 4.3 ). This enables a wider variety of processes and materials to be considered, even if one does not have access to detailed process models.

\begin{tabular}{|c|c|c|}
\hline Compromise & Material Selection & Process Selection \\
\hline $\begin{array}{l}\text { GIVEN: } \\
\text { Parametrized CAD Model } \\
\text { of Ring Gear }\end{array}$ & $\begin{array}{l}\text { GIVEN: } \\
\text { Set of alternative materials }\end{array}$ & $\begin{array}{l}\text { GIVEN } \\
\text { Set of alternative processes }\end{array}$ \\
\hline $\begin{array}{l}\text { Target values for variables } \\
\text { Target for gear weight }\end{array}$ & Desired material properties & Desired process abilities \\
\hline $\begin{array}{l}\text { FIND: } \\
\text { System Variables: } \\
\text { Diametral Pitch } \\
\text { Face Width } \\
\text { Number of Teeth }\end{array}$ & $\begin{array}{l}\text { FIND: } \\
\text { Attribute ratings for: } \\
\text { Elastic Modulus } \\
\text { Density } \\
\text { Accuracy, Finish, Build } \\
\text { Time }\end{array}$ & $\begin{array}{l}\text { FIND: } \\
\text { Attribute ratings for: } \\
\text { Cost } \\
\text { Detail Capability } \\
\text { Accuracy, Finish, Build } \\
\text { Time }\end{array}$ \\
\hline \multicolumn{3}{|c|}{ Deviation Variables: deviation of goals from target } \\
\hline $\begin{array}{l}\text { SATISFY: } \\
\text { Goals: } \\
\text { Meet target weight } \\
(0.005 \mathrm{lb} .)\end{array}$ & $\begin{array}{l}\text { SATISFY: } \\
\text { Goals: } \\
\text { Meet target elastic modulus }\end{array}$ & $\begin{array}{l}\text { SATISFY: } \\
\text { Goals: } \\
\text { Meet target cost }\end{array}$ \\
\hline $\begin{array}{l}\text { Meet target diametral pitch } \\
\text { (40 teeth/in.) }\end{array}$ & Meet target density & $\begin{array}{l}\text { Meet target detail } \\
\text { capability }\end{array}$ \\
\hline $\begin{array}{l}\text { Meet target face width } \\
(0.11 \text { in.) } \\
\text { Meet target number of } \\
\text { teeth }(48)\end{array}$ & $\begin{array}{l}\text { Meet targets on accuracy, } \\
\text { finish, build time }\end{array}$ & $\begin{array}{l}\text { Meet targets on accuracy, } \\
\text { finish, build time }\end{array}$ \\
\hline $\begin{array}{l}\text { Constraint: } \\
\text { Bending Stress }\end{array}$ & $\begin{array}{l}\text { Constraints: } \\
\text { Material / Process } \\
\text { compatibility }\end{array}$ & $\begin{array}{l}\text { Constraints: } \\
\text { Material / Process } \\
\text { compatibility }\end{array}$ \\
\hline Devir & nction: Weighted sum o & oal Deviations \\
\hline
\end{tabular}

Figure 16. Coupled Selection-Selection-Compromise Word Formulation

This problem was solved using both the LINGO optimization code and an exhaustive search in a Matlab program. For promising material-process combinations, LINGO performed an optimization to solve the compromise part of the problem, finding favorable dimensional values for the ring gear. In the Matlab program, all feasible material-process combinations were explored. 
Without going into much detail, the solution to this problem is presented in Table 7. Notice that the SLA-3500 machine and SL-5510 resin were selected as the most favorable combination of material and process. Also notice that the ring gear dimensions are further from the targets of $0.11 \mathrm{in}$. (face width) and $0.005 \mathrm{lb}$ (weight). SLA-3500 and SL-5510 were selected instead of SLA-250 and SL-5170 due to expected build time, cost, accuracy, and surface finish, even though the SLA-250/SL-5170 could produce a gear that is dimensionally closer to the production gear. This example demonstrates the trade-offs among various design and selection criteria that are frequently encountered in prototyping activities.

Table 7. Solution to the Coupled Material Selection, Process Selection, Geometric Tailoring, Ring Gear Problem

\begin{tabular}{cccc}
\hline System Variable: & Diametral Pitch & Number of Teeth & Face Width \\
Value: & 40 teeth/in. & 48 & 0.1194 in. \\
\hline Stress Constraint & \multicolumn{2}{c}{$14,395 \mathrm{psi}<14,400$ allowable } & \\
\hline Weight Goal: & Weight $=0.0054 \mathrm{lb}$ & Deviation: $0.0004 \mathrm{lb}$ & \\
\hline Goal Deviations: & Compromise & Material Selection & Process Selection \\
Value: & 0.0013 & 0.6145 & 0.591 \\
\hline $\begin{array}{c}\text { Deviation Function } \\
\text { Value: }\end{array}$ & 0.487 & & \\
& & & \\
\hline
\end{tabular}

\section{CLOSURE AND OBSERVATIONS}

In this paper, issues involved in transferring product information from design to manufacturing were described, in the context of designing and fabricating parts using Solid Freeform Fabrication (SFF) technologies. The focus of the presentation was on design methodology, rather than on the details of computable formats for representing information. Two design issues were explored: the point in the design process when the transfer occurs, and the routing of information flows through design and manufacturing activities. Three examples were presented that demonstrated information transfer and fabrication capabilities for combinations of transfer points and information flows. The main research question being investigated was: How early, and under what conditions, can design representations be transferred to manufacturing?

In answer to this question, such conditions were identified for SFF technologies in terms of information requirements and sources, including:

- For information transfer after design-for-manufacture to process planning, the designer must provide solid geometry with tolerances and 
surface finish specifications, as well as preferences for these requirements; doing so can result in very good process plans.

- For information transfer before geometric tailoring, the designer must provide parameterized solid geometry, performance requirements, fabrication process, material, and preferences among requirements are needed. Additionally, analysis models for evaluating performance requirements are needed. Utilizing a decision template with a common language (the compromise DSP was demonstrated here) enables all of this information to be transferred. The RTTB system must be able to interpret this information and solve the decision problem.

- For information transfer before materials and processes are selected, the designer must provide all the information for geometric tailoring plus suggestions for materials and processes. The RTTB system must provide materials and their properties and fabrication processes and their capabilities, in addition to selection solving capabilities.

Several unresolved issues were highlighted and lead to future research directions, including:

- Formal information models and representation formats are needed that enable general design requirements and freedoms to be communicated to manufacturers. It was suggested that the decision templates presented in this paper are promising information models.

- More complex design examples are needed to further explore the design methodology issues surrounding design-manufacture information transfer. The individual part design examples are interesting, but are lacking in the richness of assembly or product level problems.

- More comprehensive exploration of SFF technologies that includes research, not just commercial, technologies.

\section{ACKNOWLEDGMENTS}

We gratefully acknowledge NSF grant DMI-9618039. We also acknowledge the support from Rapid Prototyping \& Manufacturing Institute (RPMI) member companies and Georgia Tech for partial support for the RPMI.

\section{REFERENCES}

Allen, J. K. and Rosen, D. W. (1997) "Design Methodology Issues in a Distributed Studio for Rapid Tooling and Molded Parts," CIRP International Design Seminar Proceedings: Multimedia Technologies for Collaborative Design and Manufacturing, Los Angeles, pp. 21-28, October, 8-10, 1997. 
Bascaran, E., Bannerot, R.B., and Mistree, F. (1989) "Hierarchical Selection Decision Support Problems, in Conceptual Design," Engineering Optimization, 14: 207-238.

Connor, C.G., de Kroon, J.P., and Mistree, F. (1999) "A Product Variety Tradeoff Evaluation Method for a Family of Cordless Drill Transmissions," Proceedings ASME Design Automation Conference, paper DETC99/DAC-8625, Las Vegas, September 13-15.

Dolenc, A. and Mäkelä, I. (1994) "Slicing Procedures for Layered Manufacturing Techniques," Computer-Aided Design, 26(2):119-126.

Gerhard, J.F., Duncan, S.J., Chen, Y., Allen, J.K., Rosen, D.W., and Mistree, F. (1999), "Towards a Decision-Based, Distributed Product Realization Environment for Engineering Systems, Proceedings ASME Computers In Engineering Conference, paper DETC/CIE9085. Las Vegas, September 13-15.

Hazelrigg, G.A. (1996) Engineering Systems, Prentice Hall, Upper Saddle River, N.J.

Herrmann, A. and Allen, J. (1999) "Selection of Rapid Tooling Materials and Processes in a Distributed Design Environment," Proceedings ASME Design for Manufacturing Conference, paper DETC99/DFM-8930, Las Vegas, September 13-15.

Kulkarni, P. and Dutta, D. (1996). "An Accurate Slicing Procedure for Layered Manufacturing." Computer Aided Design, 28(9): 683-697.

Kumar, V. and Dutta, D. (1997) "An Assessment of Data Formats for Layered Manufacturing",Advances in Engineering Software, 28(3):151-164.

Lynn-Charney, C.M. and Rosen, D.W. (1999) "Accuracy Models and Their Use in Stereolithography Process Planning," accepted in Rapid Prototyping Journal.

Marsan, A., Allen, S.W., Kulkarni, P., Kumar, V. and Dutta, D. (1997). "An Integrated Software System for Process Planning for Layered Manufacturing." Proceedings from the 1997 Solid Freeform Fabrication Symposium, Austin, TX.

McClurkin, J. E. and Rosen, D. W. (1998) "Computer-Aided Build Style Decision Support for Stereolithography," Rapid Prototyping Journal, Vol. 4, No. 1, pp. 4-13.

Mistree, F., Muster, D., Shupe, J.A., and Allen, J.K., (1989) "A Decision-Based Perspective for the Design of Methods for Systems Design," Recent Experiences in Multidisciplinary Analysis and Optimization, Hampton, VA.

Mistree, F., Smith, W.F., Bras, B.A., Allen, J.K., and Muster, D., (1990) "Decision-Based Design: A Contemporary Paradigm for Ship Design," Transactions, Society of Naval Architects and Marine Engineers, 98:565-597.

Mistree, F., Hughes, O.F., and Bras, B.A., (1993) "The Compromise Decision Support Problem and the Adaptive Linear Programming Algorithm," in Structural Optimization: Status and Promise, AIAA, Washington, D.C.

Myers, R.H. and Montgomery, D.C. (1995) Response Surface Methodology: Process and Product Optimization using Designed Experiments, John Wiley \& Sons, New York.

Nemhauser, G. L. and Wolsey, L. A., (1988) Integer and Combinatorial Optimization.

Reeves, P. E. and R. C. Cobb (1997). "Reducing the Surface Deviation of Stereolithography using In-process Techniques." Rapid Prototyping Journal, 3(1):20-31.

Sabourin, E, Houser, S A, Bøhn, J H, (1996) "Adaptive Slicing using Stepwise Uniform Refinement," Rapid Prototyping Journal, 2(4):20-26.

Storti, D., Redl, C., Ganter, M., Turkiyyah, G., and Woo, T. (1999) "Encapsulated Transmission of Part Specifications for Distributed Solid Freeform Fabrication," Proceedings ASME Design Automation Conference, paper DETC99-DAC8617, Las Vegas, September 13-15.

Suh, N.P. (1990) The Principles of Design, Oxford University Press, New York.

Tata, K. (1995) "Efficient Slicing and Realization of Tessellated Objects for Layered Manufacturing," Masters Thesis, Clemson University, Clemson, SC. 
West, A.P. (1999) "A Decision Support System for Fabrication Process Planning of Stereolithography," Masters Thesis, Georgia Institute of Technology, Atlanta.

Whitney, D.E., Nevins, J.L., DeFazio, T.L., Gustavson, R.E., Metzinger, R.W., Rourke, J.M., Selzer, D.S. (1988) The Strategic Approach to Product Design, NAS, Washington, D.C.

Xu, F., Y. S. Wong, H. T. Loh, F. Y. H. Fuh and T. Miyazawa (1997). "Optimal Orientation with Variable Slicing in Stereolithography." Rapid Prototyping Journal, 3(3):76-88. 